\title{
INVESTIGACIÓN
}
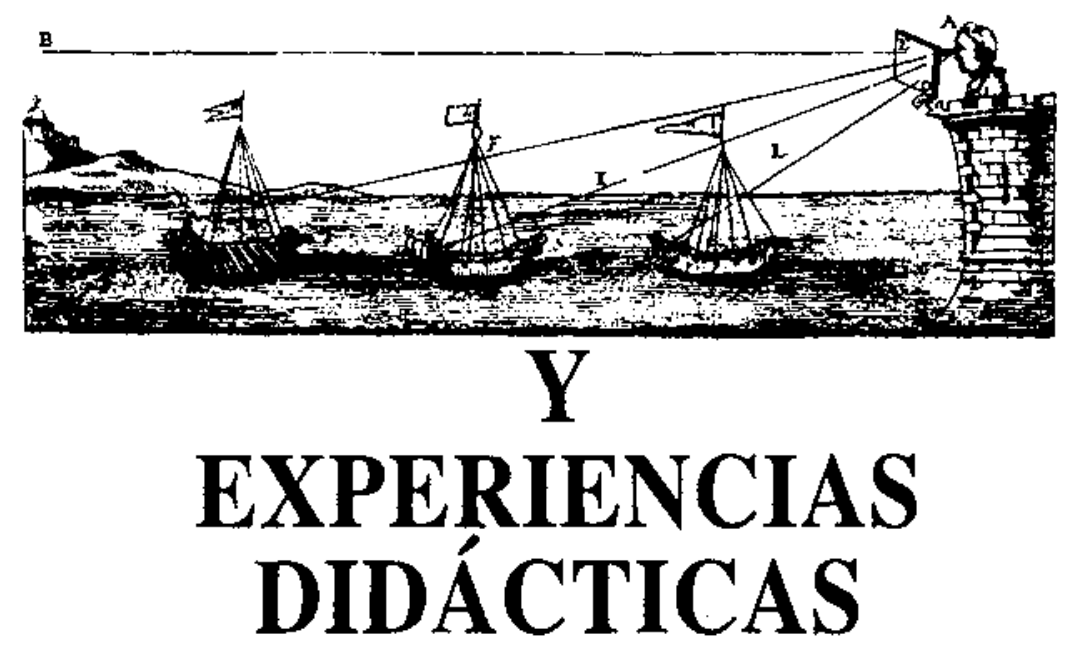

\section{UNA INVESTIGACIÓN SOBRE LOS ESQUEMAS CONCEPTUALES DEL CONTINUO. ENSAYO DE UN CUESTIONARIO}

ROMERO, C.*

IB Manuel Blancafort. La Garriga, Barcelona, cromero@ pie. xtec. es

\section{SUMMARY}

In order to survey how students conceive the idea of continuum as they begin their training on Calculus, we have established a questionnaire and an analytical approach to responses, fully rooted in networks. The theoretical framework is based on the Dreyfus and Vinner idea of "concept image», where we attune the expression uperception of a property». We have tested the questionnaire on 74 students in the range of 16-17 years old, acquiring valuable information in return about their perception on non-discrete ordering. On the other hand, we have established a draft ordered system for models\} of geometric continuum, verifying the fact that, for non-integers, the way they are written comes out to be the most representative property of the various sets of numbers. At the end, we put forth an open question about the coherence of the concept images of people with different degrees of expertise.

\section{INTRODUCCIÓN}

La modelización matemática de la naturaleza construida a partir del Renacimiento estuvo, ya desde su inicio, íntimamente ligađa a la invención y desarrollo đel cálculo infinitesimal. Esto es todavía así en nuestros días y, en 
consecuencia, una de las cuestiones más importantes que hoy nos planteamos es la de conseguir una enseñanzá eficiente del cálculo. Pero todos los conceptos básicos de la disciplina reposan sobre la noción del continuo, numérico y geométrico, y ello en dos sentidos: a) en el sentido de la construcción lógica y formal del continuo, perfectamente establecida desde los días de Weierstrass; y b) en el sentido cognitivo, que se refiere a los mecanismos de adquisición de los conceptos y métodos por parte del estudiante. Éste es el sentido por el que nos interesamos ahora.

La investigación que presentamos aquí forma parte de un proyecto más amplio, que consiste en llegar a un conocimiento exhaustivo de las concepciones -la terminología se precisa más adelante- del continuo desarrolladas por los estudiantes durante la época en que reciben instrucción básica sobre los fundamentos del cálculo. En este trabajo, sin embargo, nos referimos tan sólo a dos objetivos:

1) Construir un instrumento de investigación: un cuestionario y sus dispositivos anexos, y someterlo a ensayo.

2) Hacer una primera aproximación a las concepciones sobre el continuo de los individuos sometidos a encuesta, con la finalidad de diseñar posteriores investigaciones.

\section{EL CONTINUO COMO PROBLEMA COGNITIVO}

En la actualidad, la comunidad matemática dispone de una caracterización formal muy satisfactoria del continuo. Respecto del continuo numérico, la obra de Weicrstrass, Cantor, Méray y Dedekind mantiene su plena vigencia', mientras que, entre otros, Hilbert (18991930), al enunciar axiomas explícitos de continuidad para la recta, caracterizaba el continuo geométrico. Además, se establecía la equivalencia lógica de ambas perspectivas en la forma que, tres siglos antes, ya habian propuesto Descartes y Fermat ${ }^{2}$.

La caracterización lógico-formal del continuo es, pues, un camino posible de aproximación al problema y, por tanto, puede constituirse en fundamento de un cierto modelo de enseñanza. Pero resulta muy dudoso que el conocimiento de los individuos sobre el continuo pueda construirse sobre la caracterización lógico-formal, en la manera en la que se suele aprender, por ejemplo, Ia teoría de grupos. En efecto: un grupo se define como un sistema sujeto a determinados axiomas, mientras que, en una construcción formal de los números reales, debido a que los estudiantes ya los conocen con más o menos vaguedad, sí se consigue mostrar la existencia de un cuerpo completamente ordenado, pero no que los números reales formen una tal estructura (Steiner, 1984).

Por otro lado, cuando, por las razones que sean, se procura evitar a los estudiantes una introducción axio- mática o constructiva de los números reales, suele apelarse a una soi-disant "visión intuitiva» de las propiedades de la línea recta. El mismo Dedekind expresa esto en forma paradigmática cuando reclama la conveniencia de acudir a las evidencias geométricas usi no se quiere perder demasiado tiempo" (Dedekind, 190, p. 1). Pero entonces se están admitiendo dos hipótesis implícitas: a) que el continuo geométrico es un dato universal de la intuición, fácilmente asequible a los individuos; y b) que la conexión números-geometría es, del mismo mocto. intuitiva, es decir, aprehensible inmediata y universalmente, sin la necesidad de que medien elementos conceptuales, los cuales pertenecen al modo de pensamiento discursivo. Sin embargo, la experimentación que presentamos mostrará que, cuando menos desde el punto de vista didáctico, hay indicios claros de que estas dos hipótesis son falsas.

\section{EL MARCO TEÓRICO DE NUESTRO TRABAJO}

En una primera aproximación, por tanto, nuestro problema consiste en estudiar la intuición del continuo de los individuos. El término «intuición» tiene una sólida tradición en la filosofía y ya, en la Grecia clásica, se distingúa el modo de conocimiento intuitivo (vónolc) mediante el cual las realidades se ven directamente y las verdades se aprehenden sin ningún olemento conceptual mediando entre el intelecto y la cosa intuida, del modto de conocimiento discutsivo ( $\mathrm{L} \alpha \mathrm{vol} \alpha$ ). Kant contrapone la intuición al concepto, pero Descartes la contrapone a la deducción. De hecho, la acepción cartesiana es la más difundida entre la comunidad matemática, donde se pervierte así: «intuitivo» significa «carente de rigor»?.

Pero el concepto filos6fico de "intuición», en cualquiera de sus variantes, es manifiestamente insuficiente para los propósitos de una investigación como la nuestra porque, entre otras razones, no contiene ninguna referencia a la conexión entre la «cosa intuida» y el «intelectom. Por otra parte, estudios epistemológicos recientes insisten en el hecho de que el continuo, lejos de ser uni determinación intuitiva simple, es un producto muy elaborado de la meditación ontológica y de la conceptualización matemática (Caveing, 1982).

Alternativamente, diversos autores han usado diferentes términos: "concepción», «imagen mental», etc. $y$, en sus trabajos, han definido los significados con grados de precisión diversos. Nosotros, con el propósito de delimitar bien el objeto de nuestro estudio, hemos acudido a la noción de «concept image»-que traducimos por "esquema conceptual»... de Vinner (1983), Vinner y Dreyfus (1989), Dreyfus (1990), Tall (1991a) y Vinner (1991). En esta elección, así como en la traducción del término, hemos seguido a Azcárate (1990), y la justiftcación principal reside en que la definición delimita bien el concepto $y$, además, se enmarca plenamente en la perspectiva constructivista de la ciencia cognitiva. 
La noción de «esquema conceptual» («concept image») nace para distinguir entre el modo en que cada individuo piensa un concepto y la definición formal («concept definition», en los propios términos de los inventores de tal noción) de ese mismo concepto:

«Todos los conceptos matemáticos, excepto los más primitivos, tienen definiciones formales. Muchas de estas definiciones se dan a los estudiantes de secundaria o de universidad en un momento u otro. El estudiante, por otra parte, no usa necesariamente la definición cuando decide si un objeto matemático dado es o no un ejemplo del concepto. En la mayoría de los casos, la decisión se toma en base a un esquema conceptual, esto es, el conjunto de todas las imágenes mentales asociadas al nombre del concepto en la mente del estudiante, junto con todas las propiedades que lo caracterizan (por imagen mental entendemos cualquier tipo de representación: icónica, simbólica, diagramas, gráficos, etc.). El esquema conceptual es el resultado de su experiencia con ejemplos y contraejemplos del concepto. Resulta que el conjunto de los objetos matemáticos que el estudiante considera como ejemplos del concepto no es necesariamente idéntico al conjunto determinado por la definición. Si estos dos conjuntos no coinciden, el comportamiento del estudiante puede ser diferente al que el profesor espera» (Vinner y Dreyfus, 1989).

Hemos hecho la hipótesis, que creemos coherente con la noción de esquema conceptual, de que, más allá de ta definición de un concepto, los individuos también tratan con sus «propiedades», no a partir de sus implicaciones lógicas, sino en relación con sus propios esquemas conceptuales sobre el concepto: un mismo enunciado verbal de una propiedad puede impeler individuos con esquemas conceptuales distintos a acciones diferentes y, muchas veces, incoherentes entre sí. Usaremos el término "percepción de una propiedad» para denotar esa interacción entre el enunciado de tna propiedad y el esquema conceptual del individuo que la pone en juego.

\section{PERSPECTIVA HISTÓRICA}

La noción de continuo geométrico quedó bien establecida en la definición de «magnitudes proporcionales» de Eudoxo, en los Elementos, $V$, def. 5. Esta definición tan sólo es geométrica y se inscribe en el paradigma euclidiano lógico-deductivo, pero se fundamenta en la noción euclídea de «magnitud», la cual, en nuestro sentido, no es «numérica».

En cambio, el continuo numérico no se percibió hasta mucho más tarde. La noción de continuo numérico es el resultado de una larguísima evolución, de la cual tenemos las primeras noticias en la matemática babilónica, y que penetró en Occidente con toda su fucrza a partir de la generalización del cálculo con fracciones decimales, cuando se publica La Disme de Simon Stevin en 1585. F́ste es un modo de pensamiento algorítmico que ignora la fundamentación lógica. Entonces, para fundamentar los usos de los números, aparecen los números reales, los cuales constituyen la entidad matemática que coagula la noción, más o menos indefiniđa, paramatemática (en el sentido de Chevallard [1991]), de «magnitud».

Intentamos la síntesis de los dos tipos de continuo por medio del concepto de recta graduada ${ }^{\downarrow}$. Para la magnitud paradigmática, la longitud, se pide que la recta graduada contenga puntos suficientes para que la unidad, definida previamente, mida cualquier otra longitud establecida por cualquier otro camino. El problema cognitivo surge cuando este «otro camino" cs numérico, fruto de algoritmos de cálculo: se habla entonces del «fracaso de la unión de la geometría y los números» (Cornu, 1991, pp. 159-160) y es en el seno de este conflicto donde encuentra su justificación nuestro estudio, reforzado por el hecho de que, en la enseñanza no universitaria de las matemáticas, se suele primar el modo de pensamiento algorítmico, como consecuencia seguramente de la propia dinámica de la adquisición y desarrollo de los conceptos (Hiebert y Wearne, 1986 y Sfard 1991).

\section{BIBLIOGRAFÍA RELACIONADA CON NUESTRO TEMA}

Los trabajos que hemos podido consultar y que, de una forma u otra, se relacionan con cl tema que nos ocupa, se pueden clasificar en tres grupos:

a) En torno a la concepción de recta geométrica. En Mansfield (1985) se comparan las concepciones respecto de puntos, líneas y sus relaciones, de estudiantes de $7^{\circ}$ grado con las de estudiantes de un college. Se muestran paradigmas de representación mental que encontraremos también en nuestra investigación: puntos como discos pequeños, líneas como cintas, etc.

b) En torno a la adquisición de los conceptos de límite y de derivada. Constituye el grupo más abundante y en casi todos ellos se explota a fondo la noción de cobstáculo epistemológico, acuñada à partir de la clásicás pro. puesta de Bachelard (1938). Citemos aquí los trabajos de Cornu (1980, 1982, 1991), Robinet (1983) y Sierpinska $(1985,1987)$. El elemento común a todas estas exposiciones que ha suscitado nuestro interés es la descripción de ciertos obstáculos epistemológicos, que vemos ligados a las diversas concepciones del continuo, aunque sus autores no hagan mención explícita de ello.

c) En torno a la adquisición de conceptos ligados a los números no enteros. La mayoría de ellos se reficren a problemas didácticos correspondientes a etapas de la enseñanza previas a la que aquí nos ocupa. Citemos Brousseau (1981), Hiebert y Wearne (1986) y Brousseau (1983), además de breves notas İiebeck (1985) y Fowler (1985).

Más acordes con nuestros objetivos son Steiner (1984), también en una nota breve, y Robinet (1986), que, en buena medida, trata el mismo problema que planteamos en nuestro trabajo. 


\section{NUESTRO CUESTIONARIO}

Este trabajo es una investigación sobre esquemas conceptuales y de percepciones de propiedades $\mathrm{y}$, por to tanto, de datos cualitativos. En consecuencia, tanto el instrumento de investigación (el cuestionario) como los métodos de análisis han sido diseñados para ser capaces de tratar con ese tipo de datos.

Para elaborar el cuestionario, hemos examinado la experiencia descrita en Robinet (1986) y, por otra parte, hemos hecho ensayos previos del funcionamiento de las preguntas que lo componen mediante entrevistas a personas de edades diversas, para las que las matemáticas son un componente esencial en su profesión. Como resultado de ello y de las consideraciones teóricas que ya hemos expuesto antes, hemos adoptado el criterio de centrar la investigación en los modelos mentales y en la percepción de las propiedades de las magnitudes continuas, prescindiendo de incidir en distinciones más académicas de tipos de números (números naturales, enteros, racionales, reales, complejos, etc.).

Las preguntas de nuestro cuestionario fueron contestadas por 74 estudiantes de $3^{\circ}$ de BUP, y son las siguientes:

1) Con la pretensión de investigar las percepciones de las propiedades y características de cada tipo de números, hemos propuesto una tarea que hace poner en juego estas percepciones y que consiste en hacer que los individuos busquen y establezcan criterios de clasificación de números. El conocimiento de las percepciones de las propiedades de los números ha de proporcionar información importante acerca de las posibilidades que tienen los individuos para establecer nexos conceptuales entre los continuos numérico y geométrico.

A cada individuo encuestado le pedimos, pues, que clasifique los siguientes números que encuentra en un cuadro como éste, distribuidos al azar:

$$
\begin{aligned}
& \text { 9. } 217 \quad 735-3 \frac{3}{4} \quad \frac{2}{7} 0.75 \quad 6.325 \quad 3.1416 \\
& 5.3 \times 10^{4} \quad 0.1919 \ldots \quad 2.9999 \ldots-\frac{5}{4}-7.75 \\
& -4.3333 \ldots \sqrt{2} 1.1010010001000 \ldots 1.23456 \ldots \pi \sqrt{-4}
\end{aligned}
$$

Las consignas de actuacion especifican que hay que evitar criterios escolásticos ${ }^{5}$, que se especifiquen claramente los criterios empleados y, para nuestra validación de las respuestas, que se muestren dos ejemplares más de números que no estén en la lista, correspondientes a cada uno de los criterios de clasificación. Notemos además que el conjunto de números que se propone contiene "parejas ocultas» (como $3 / 4$ y $0,75,03,1416$ y $\pi$, etc.) y el número $\sqrt{-4}$ que está fuera del ámbito de las «magnitudes" en el sentido "griego".

2) La segunda pregunta pide la descripción de un objeto continuo -una recta- y la hemos planteado bajo la hipótesis de que los esquemas conceptuales de los objetos geométricos se construyen principalmente a partir de experiencias de visualización. La hemos formulado así:
«Imagina que dispones de un microscopio de gran potencia. Más aun, que puede aumentar los objetos tanto como tá quieras. Ahora enfocas un fragmento de una recta. Describe lo que ves y lo que ocurre a medida que vas aumentando la potencia del microscopio. ¿Puedes describir lo que ves cuando el microscopio tiene aumento infinito?"

Esta pregunta es una reformulación de la cuarta del cuestionario de Robinet (1986), convenientemente modificada para permitir captar el aspecto dinámico de la experiencia imaginaria que se propone, así como para poder investigar sobre la noción de infinito de los individuos. Nuestro máximo interés se concentra en las descripciones de los individuos de lo que wocurre» mientras la potencia del microscopio va aumentando.

3) En la tercera pregunta proponemos una tarea de manipulación de un objeto acotado y completo; pedimos que, mediante un instrumento de corte eperfecto», una cuerda de un metro de longitud (la imagen tradicional de un segmento) se someta al siguiente proceso indefinido:

«Con las tijeras, cortar la cuerda en dos trozos, iguales o no. Tirar uno de los fragmentos a la basura. Con las tijeras, cortar la cuerda que nos queda en dos trozos, iguales o no. Tirar uno de los fragmentos a la basura. Con las tijeras, cortar...”

Evidentemente, antes le hemos aclarado al individuo encuestado que, así como las tijeras son "perfectas", la cuerda es una "cuerda matemática», y nos interesaremos por el significado que el individuo pueda dar a esta expresión.

Pedimos entonces que el individuo explique si cree que el proceso puede continuar indefinidamente y que nos describa, según la opción que haya elegido, qué tenía en sus manos antes del último corte posible, o bien, después de infinitos cortes. Aquí nos proponemos dilucidar el modelo de recta que se ha usado, así como la percepción de la propiedad de completitud.

\section{MÉTODO DE ANÁLISIS DE LOS DATOS}

Para compilar las respuestas, hemos hecho un uso intensivo de la técnica de redes sistémicas, «network analysis» (Bliss et al., 1979), bajo la hipotesis de que es posible tratar los esquemas conceptuales como conjuntos de opciones de modelos de representación en el seno de entornos semánticos diversos, los cuales admiten posibilidades variadas de este tipo de opciones.

En el método de las redes sístémicas, cada respuesta se contempla como una opción que tiene significado porque es una configuracion particular de una red estructurada de opciones interdependientes. Cada configuración posible de elecciones es un paradigma (de acuerdo con la terminología de Bliss) y cada uno de los significados corresponde a uno de los paradigmas posibles. La vali- 
dez del método se apoya en la hipótesis de que el significado relevante de las opciones disponibles en cada entorno no reside en la relación entre el modelo de representación y el objeto representado, sino en el contraste con otras opciones posibles del mismo entorno. Desde un punto de vista utititario (quizá el del profesor), el significado relevante de una cierta representación mental de un concepto es la acción, la respuesta que un individuo elabora al tratar de resolver una situación problemática relacionada con el concepto. Pero al investigador, aquello que le interesa dilucidar es el porqué de esta acción y no de otra dentro de un cierto abanico de posibilidades, en correspondencia con una elección concreta en el conjunto de representaciones posibles.
Una red sistémica consiste, pues, en una red estructurada de opciones independientes en la cual se distinguen aspectos (simultaneidad) y clases (donde una opcion excluye a las demás). La red, hasta aquí, es un esquema abstracto que se materializa asociando a cada uno de sus nudos un "código», es decir, un elemento de lenguaje ordinario, el cual, usualmente, no es sino una transcripción de la respuesta más representativa. Entonces la red actúa como un lenguaje artificial mediante el cual el analista dice lo que ve y cada respuesta se simboliza por el conjunto de símbolos de los nudos de la red que la representan.

Así, por ejemplo, veamos una muestra de cómo se insertan dos respuestas en una red:

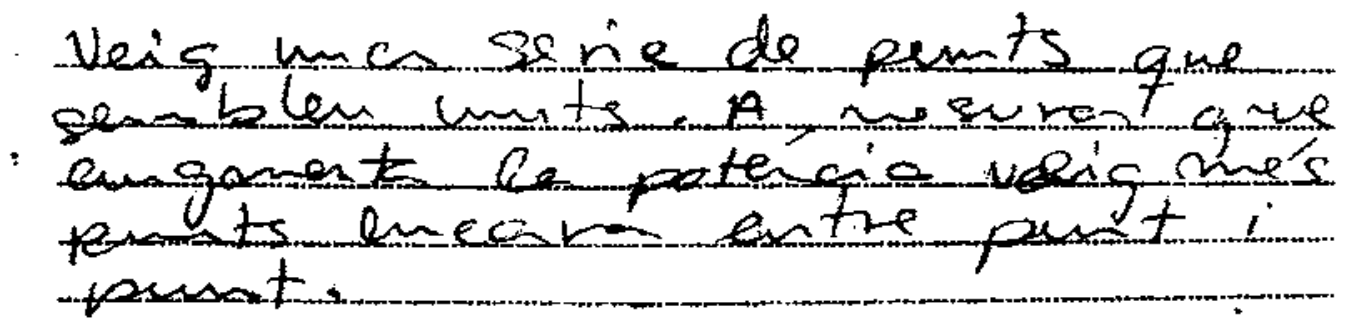

«Veo una seric de puntos que parecen unidos: A medida que aumenta la potencia veo aún más puntos entre punto y punto.»

Respuesta a la segunda pregunta. Individuo 67:

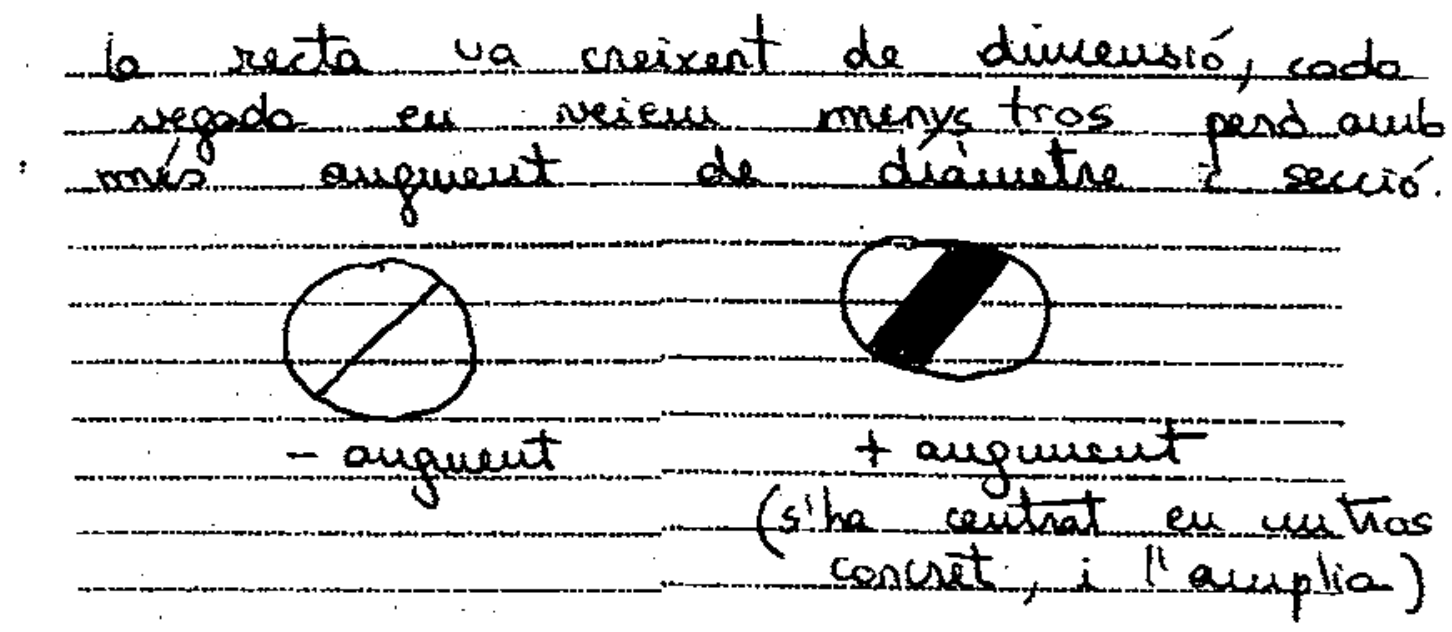

«La recta va creciendo en dimensión; cada vez vemos una parte menor, pero com más aumento de diámetro y de sección,» 
El individuo 33 ve elementos (puntos), mientras que el individuo 67 ve un objeto, pero no elementos en su seno. Otros individuos vieron marcas de medida, como en una regla, otros vieron números... En el fragmento de la red sistémica (Fig. I) que mostramos (la cual ha sido elaborada a partir de la consideración de todas las respuestas), la Iocalización del individuo 33 corresponde a Ios nudos $C 4 E 2, C 4 F 3$, y la del individuo 67 al nudo C32.

Figura 1

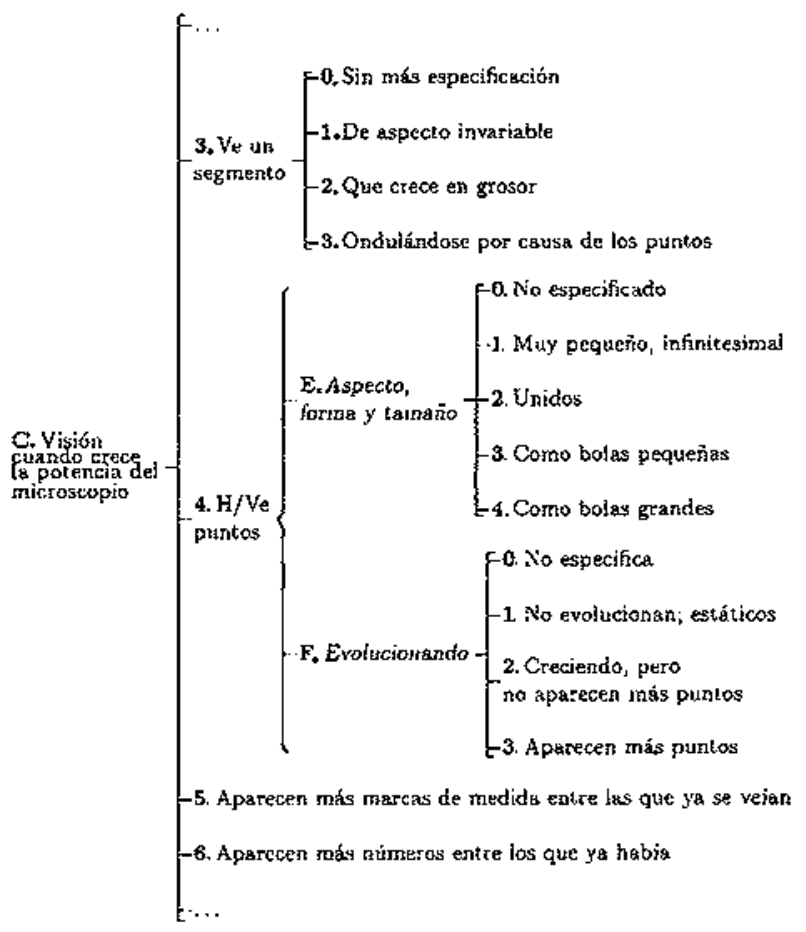

Figura 2
A. P'recisamente, porque son números
B. Por su paridad (par/imper)
C. Por su signo $(+/-)$
D. Por su tipo (N, Z, Q, etc.)
E. Porque no pertenecen a la recta gradusda
F. Pot el modo en que se escriben
G. Por su relación con otros campos u objetios
YI. Por su relación con la nación de magnitud
I. Por su grafo de accesibifidad o perceptibilided
3. Por su relación con otros elementos del conjunto que se ha formado
K. Por sus valores afectivos
L. Por su tamaio

\section{DATOS RECOGIDOS. RESULTADOS}

1) Al estudiar las respuestas a la primera pregunta del cuestionario hemos hecho un análisis exhaustivo de los siguientes puntos: $a$ ) cuáles y cuantos grupos de números se hicieron y de qué longitud; b) cuáles fueron los criterios de clasificación usados; c) cómo se clasificó cada uno de los números propuestos; y d) las «parejas ocultas».

La figura 2 muestra los criterios de clasificación de números que propusieron los individuos encuestados, y la figura 3 es la tabla de frecuencias para cada uno de los números propuestos.

Hay que remarcar la variación de cifras que aparce cuando el tipo de números pasa de los naturales a los irracionales: un hecho se pone de manifiesto cuando se comparan las cifras correspondientes al criterio $D$ (por su tipo: $N, Z, Q$, etc.) y las del criterio $F$ (por el modo en que se escriben): es que las frecuencias del primero caen tan rápidamente como crecen las del segundo. Más adelante comentaremos este aspecto.

Figura 3

\begin{tabular}{|c|r|r|r|r|r|r|r|r|r|r|r|r|}
\hline Numeros: & A & $\mathbf{B}$ & $\mathbf{C}$ & $\mathbf{D}$ & $\mathbf{E}$ & $\mathbf{F}$ & $\mathbf{G}$ & $\mathbf{H}$ & $\mathbf{I}$ & $\mathrm{J}$ & $\mathrm{K}$ & $\mathbf{L}$ \\
\hline 9 & 1 & 7 & 32 & 48 & 0 & $\mathrm{II}$ & 0 & 1 & 4 & 3 & 7 & 0 \\
217 & 1 & 7 & 33 & 49 & 0 & 11 & 0 & 1 & 4 & 0 & 7 & 0 \\
735 & 1 & 6 & 32 & 49 & 0 & 11 & 0 & 1 & 4 & 2 & 7 & 0 \\
-3 & 1 & 2 & 44 & 39 & 0 & 12 & 0 & 2 & 7 & 3 & 4 & 0 \\
$3 / 4$ & 1 & 0 & 24 & 17 & 0 & 47 & 0 & 1 & 5 & 3 & 4 & 0 \\
$2 / 7$ & 1 & 0 & 20 & 17 & 0 & 49 & 0 & 1 & 4 & 0 & 4 & 0 \\
0.75 & 0 & 0 & 22 & 13 & 0 & 41 & 0 & 1 & 9 & 3 & 3 & 1 \\
6.325 & 1 & 0 & 22 & 13 & 0 & 38 & 0 & 1 & 8 & 1 & 2 & 1 \\
3.1416 & 1 & 0 & 20 & 11 & 0 & 36 & 2 & 1 & 8 & 9 & 2 & 0 \\
$5.3 \times 10^{4}$ & 1 & 1 & 20 & 21 & 0 & 37 & 0 & 2 & 5 & 0 & 5 & 2 \\
$0.1919 \ldots$ & 1 & 0 & 17 & 10 & 0 & 55 & 0 & 1 & 8 & 0 & 2 & 0 \\
$2.9999 \ldots$ & 1 & 0 & 15 & 11 & 0 & 54 & 0 & 1 & 7 & 0 & 1 & 0 \\
$-5 / 4$ & 1 & 0 & 32 & 17 & 0 & 45 & 0 & 1 & 6 & 0 & 3 & 0 \\
-7.75 & 1 & 0 & 37 & 16 & 0 & 37 & 0 & 1 & 9 & 0 & 3 & 1 \\
$-4.333 \ldots$ & 0 & 0 & 29 & 11 & 0 & 53 & 0 & 2 & 7 & 0 & 4 & 0 \\
$\sqrt{2}$ & 1 & 0 & 13 & 16 & 0 & 44 & 0 & 1 & 8 & 0 & 3 & 0 \\
$1.1010010 \ldots$ & 1 & 0 & 12 & 9 & 0 & 53 & 0 & 1 & 9 & 0 & 3 & 0 \\
$1.2345678 \ldots$ & 1 & 0 & 13 & 10 & 0 & 52 & 0 & 1 & 8 & 0 & 3 & 0 \\
$\pi$ & 1 & 0 & 9 & 12 & 0 & 33 & 6 & 1 & 9 & 8 & 6 & 0 \\
$\sqrt{-4}$ & 1 & 0 & 3 & 30 & 1 & 26 & 0 & 1 & 15 & 0 & 7 & 0 \\
\hline
\end{tabular}

Antes, mostremos algunas respuestas:

Individuo 26

Grupo de números: $\{3.1416, \pi\}$ Criterio: Jl. 


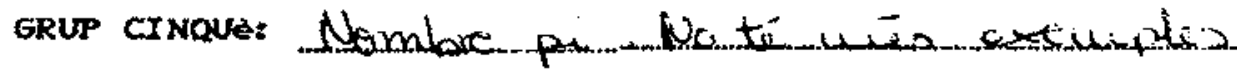

Individuo $I$

Grupo de números: $\{3.1416, \pi\}$ Criterios: F1B22, F3.

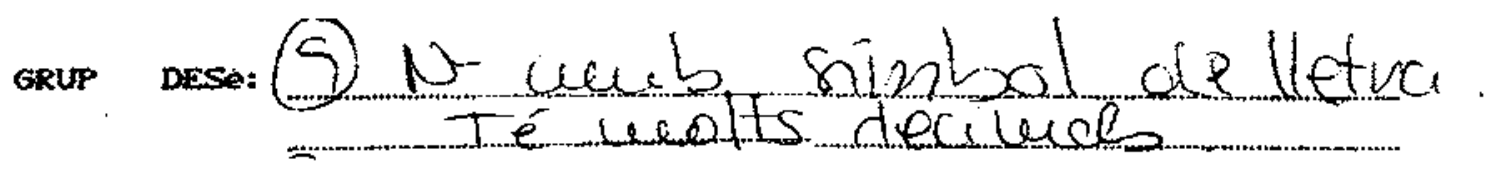

«Número con símbolo de letra. Tiene muchos decimales."

Individuo 11

Grupo de números: $\{\pi\}$ Criterio: 15 .

Grupo de números: $\{1.1010010001 \ldots, 1.234567891011 \ldots\}$ Criterios: H1B232, 12

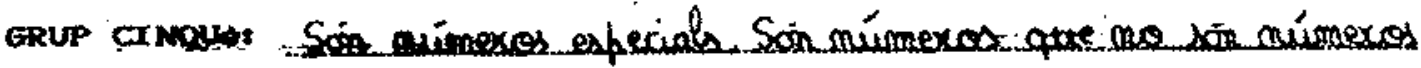

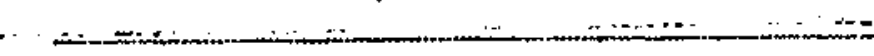

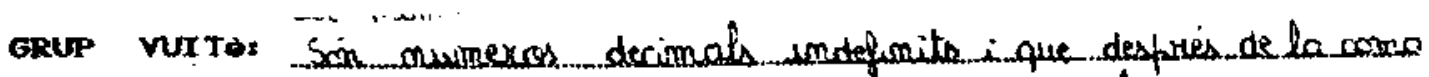
no pouten sumpre el mateix isumeto ...pro són esfecials.

"Son números especiales. Son números que no son números."

«Son números decimales indefinidos y que después de la coma no llevan siempre el mismo número, pero son especiales.»

Individuo 49

Grupo de números: $0.75,6.325,3.1416,0.1919 \ldots, 2.9999 \ldots, \quad$ I. $23456789101112 \ldots \pi$ \}

Criterios: F1B2, 12\}.

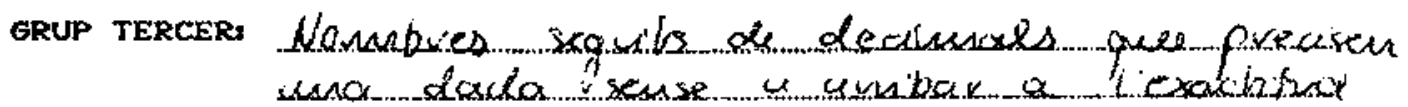

«Números seguidos de decimales que precisan un dato sin llegar a la exactitud.»

Observemos ahora el recuento de frecuencias de aparición de algunas "parejas ocultas" agrupadas según criterios de clasificación (de $\mathrm{A}$ a L L, Fig, 4). La evidencia abrumadora de que, para los individuos encuestados, la característica más significativa de los números no enteros es su forma de escritura es el resultado más llamativo de nuestro estudio: el recuento del número de veces que aparece clasificado 
cada uno de los números no enteros de la lista según cada criterio proporciona máximos significativos a la forma de escritura en detrimento de otros criterios. Así, pues, números con escrituras diferentes resultán ser números de especies distintas. El estudio de las «parejas ocultas» reafirma todavía más esta con- clusión: los números 9 y 735 aparecieron 116 veces asociados, mientras que 9 y $5,3 \times 10^{4}$ se asociaron en 49 casos, y 9 y $2,9999 \ldots$ tan sólo en 4 . Pero, si sucede que estos números están ligados a algún uso específico $(3,1416$ y $\pi)$, entonces se les considera «iguales».

\begin{tabular}{|c|c|c|c|c|c|c|c|c|c|c|c|c|c|}
\hline Parejas ocuitas: & $\mathrm{A}$ & B & $\mathrm{C}$ & DT & $E$ & $F$ & $\mathrm{G}$ & $\mathrm{H}$ & & $3 \pi$ & & & Totales: \\
\hline 9 y 217 & 1 & 7 & 35 & 50 & 0 & 10 & 0 & 1 & 4 & $\overline{0}$ & 7 & 0 & 115 \\
\hline 9 y 735 & 1 & 6 & 35 & 50 & 0 & 10 & 0 & 1 & 4 & 2 & 7 & 0 & 116 \\
\hline 217 y 735 & 1 & 6 & 35 & 51 & 0 & 11 & 0 & 1 & 4 & 0 & 7 & 0 & 116 \\
\hline 9 y $5.3 \times 10^{4}$ & 1 & 0 & 14 & 19 & 0 & 9 & 0 & 1 & 3 & 0 & 2 & 0 & 49 \\
\hline 217 y $5.3 \times 10^{4}$ & 1 & 0 & 14 & 20 & 0 & 9 & 0 & 1 & 3 & $0:$ & 2 & 0 & 50 \\
\hline 735 y $5.3 \times 10^{4}$ & 1 & 0 & 14 & 20 & 0 & 10 & 0 & 1 & 3 & 0 & 2 & 0 & 51 \\
\hline $3 / 4$ y 0.75 & 0 & 0 & 12 & 10 & 0 & 16 & 0 & 1 & 5 & 3 & 2 & 0 & 49 \\
\hline 3.1416 y $\pi$ & 1 & 0 & 5 & 3 & 0 & 10 & 2 & 0 & 3 & 8 & 1 & 0 & 33 \\
\hline$\sqrt{2}$ y $\sqrt{-4}$ & 1 & 0 & 2 & 2 & 0 & 19 & 0 & 1 & 3 & 0 & 2 & 0 & 30 \\
\hline 9 y $2.9999 \ldots$ & 1 & 0 & 2 & 1 & 0 & 1 & 0 & 0 & 0 & 0 & 0 & 0 & 5 \\
\hline 217 y $2.9999 \ldots$ & 1 & 0 & 2 & 1 & 0 & 0 & 0 & 0 & 0 & 0 & 0 & 0 & 4 \\
\hline 735 y $2.9999 \ldots$ & $I$ & 0 & 2 & 1 & 0 & 0 & 0 & 0 & 0 & 0 & 0 & 0 & 4 \\
\hline$\sqrt{2}$ y $1.101001 \ldots$ & 1 & 0 & 5 & 8 & 0 & 15 & 0 & 1 & 3 & 0 & 0 & 0 & 33 \\
\hline$\sqrt{2}$ y $1.23456 \ldots$ & 1 & 0 & 5 & 9 & 0 & 16 & 0 & 1 & 3 & 0 & 0 & 0 & 35 \\
\hline$\sqrt{2}$ y $\pi$ & 1 & 0 & 8 & 11 & 0 & 16 & 0 & 1 & 4 & 0 & 1 & 0 & 42 \\
\hline $1.101001 \ldots$ y $1.23456 \ldots$ & 1 & 0 & 10 & 7 & 0 & 46 & 0 & 1 & 7 & 0 & 3 & 0 & 75 \\
\hline $1.101001 \ldots$ y $\pi$ & 1 & 0 & 6 & 8 & 0 & 21 & 0 & 1 & 4 & 0 & 1 & 0 & 42 \\
\hline $1.23456 \ldots$ y $\pi$ & 1 & 0 & 6 & 9 & 0 & 19 & 0 & 1 & 4 & 0 & 1 & 0 & 41 \\
\hline $3 / 4 \times 2 / 7$ & 1 & 0 & 17 & 16 & 0 & 40 & 0 & 0 & & & & U & 80 \\
\hline
\end{tabular}

2) Nuestro estudio de las respuestas a la segunda pregunta del cuestionario consistc en analizar exhaustivamente: a) los contextos semánticos de las respuestas, sea el contexto "realístico», "físico» o «matemático»; b) los modelos mentales de la estructura de la línea recta, así como su variación durante los tres tiempos (mirar la línea recta a través del microscopio, aumentar la potencia y llegar a potencia infinita) de la experiencia imaginaria que proponíamos; y c) las relaciones entre los modelos mentales y la percepción de la propiedad del orden no discreto.

Ya hemos presentado antes algunos ejemplos de respuestas. Ahora hemos de decir que el análisis de las respuestas a esta pregunta permite hacer una primera clasificación de los esquemas conceptuales de los individuos correspondientes al concepto de línea recta. En efecto, aparte de un $6,75 \%$ marginal que no pudieron «jugar el juego» de imaginación que se les proponía y que calificamos de «realistas ingenuos», el resto de individuos encuestados mostró, con grados de tensión diversos, la interacción entre sus percepciones de las propiedades abstractas de la recta como econstrucción mental pura» y sus «imágenes» de la recta como «objeto físico». La recta se percibe, o bien como una especie de cinta, o como un conjunto de puntos que, con frecuencist, son discos o pequeñas esferas.

Hemos hecho un estudio de estabilidad de las imágenes durante los tres tiempos de la experiencia imaginaria: si bien al empezar a mirar, el $47,3 \%$ ve la recta como un todo y el $29,7 \%$ ya percibe elementos, al llegar a aumento infinito, la proporciones respectivas son del $20,3 \%$ y $37,8 \%$. En contraposición al «realismo ingenuo» tenemos, pues, una «abstracción ingenua».

Por otra parte, las visiones de la estructura fina de la recta nos permiten establecer perfiles de individuos 
«continuistas» y de «atomistas». Hay que hacer notar que la estructura de orden no discreto no aparece en ninguna de las descripciones de los continuistas, mien* tras que, entre los atomistas, se reparten en porcentajes iguales aquéllos que no parecen tener la propiedad del orden no discreto en su esquema conceptual y los que, en sus respuestas, muestran evidencia de una cierta lucha por dar una imagen coherente con esta propiedad.

Finalmente, las respuestas nos dan indicios, aunque muy débiles, para dilucidar qué tipo de infinito aceptan los individuos. El resumen de lo anterior es el árbol de la figura 5. En él no aparecen porcentajes relativos à la percepción del tipo de infinito porque, como acabamos de decir, los indicios són demasiado débiles para poder cuantificar con significado.

3) De las respuestas a la tercera pregunta del cuestionario hemos analizado exhaustivamente: a) los contextos semánticos de las respuestas, en la misma forma que en el estudio de la segunda pregunta; b) las descripciones que los individuos hacen del residuo que queda en sus

Figura $S$

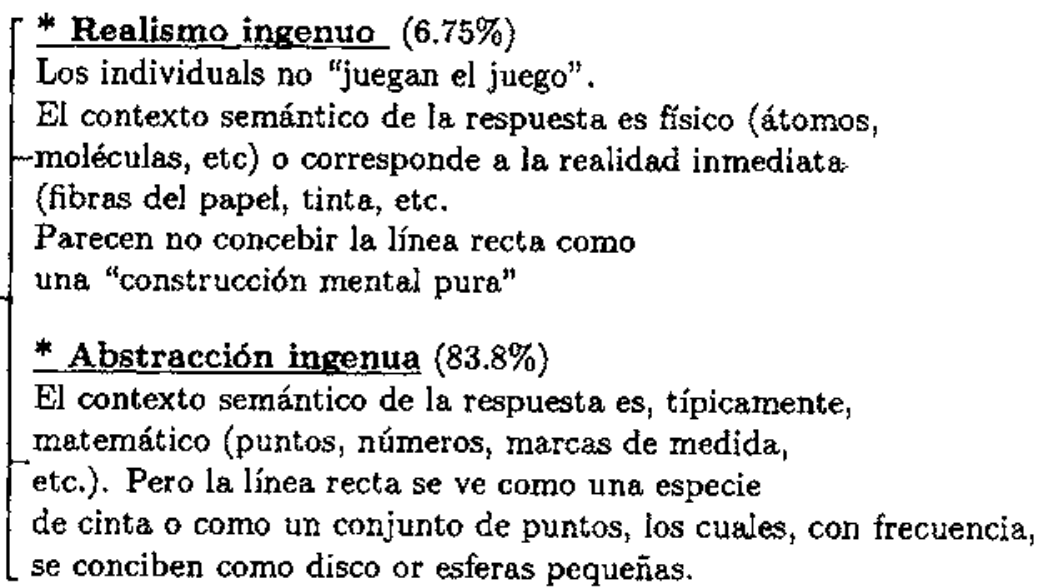

* Continuistas (Del $47.3 \%$ en el primer tiempo, al $20.3 \%$ en el tercer tiempo de la "experiencia")

Los individuos ven la línea recta como un todo, sin reconocer elementos en ella.

No hay ninguna dificultad con la estructura de orden no discreto: los individuos la ignoran

de la

linea

recta
*Atomistas (Del $29.7 \%$ en el primer tiempo, al $37.8 \%$ en el tercer tiempo de la "expe"riencia") Los individuos ven elementos en la línea recta, más o menos estructurados.

\section{* No se incluye el orden no discreto - Los individuos ignoran la estructura de orden no discreto y sus eventuales difficultades \\ * Se incluye el orden no discreto Los individuos luchan para construir su propia -imagen de la línea recta como conjunto de elementos coherentemente con la más o menos conocida propiedad del orden no discreto.}

$$
\text { -Inflnito }\left\{\begin{array}{l}
\frac{* \text { Potencialistas }}{\text { Las respuestas de los individuos muestran el infinito }} \\
\text { como un proceso sin fin, dinámico. } \\
\begin{array}{l}
\text { * Actualistas } \\
\text { Las respuestas de los individuos muestran el infinito } \\
\text { en forma estática. Es alcanzable. }
\end{array}
\end{array}\right.
$$


manos al final do ese otro experimento imaginario y sus justificaciones; y c) las percepciones de procesos ilimitados.

Ahora el $21 \%$ de los individuos afirma que el proceso de subdivisión es necesariamente finto, mientras que el $58,1 \%$ lo reconoce como necesariamente indefinido, pero no aparece ninguna correlación significativa con el ámbito semántico de la respuesta, el cual no ha resultado ser un aspecto discriminador de respuestas. Más aun: tampoco hemos encontrado correlación significativa con los modelos de recta que se obtienen del estudio de la segunda pregunta y que hemos establecido en el cuadro anterior. En cambio, hemos hallado una correlación significativa al $95 \%$ entre la opción de respuesta y la necesidad, sentida por los individuos, de explicarse respecto de las causas del resultado que obtienen en el proceso de división de la cuerda: quienes afirman que el proceso será indefinido no explican el porqué de esto, mientras que los que creen que el proceso será siempre fínito se ven impelidos a explicario. El análisis de este hecho es difícil, pero nos arriesgamos a formular una hipótesis, que habría que comprobar por otros medios y que niega las «hipótesis de la intuitividad inmediata del continuo»: los diferentes esquemas conceptuales del continuo no son, en general, más que un agregado muy inconexo de imágenes mentales y de propiedades adquiridas de maneras muy diversas, entre las cuales encontramos la intimidación (Lang, 1985) y, hasta cierto punto, esto se ve confirmado por la experiencia de Fischbein y otros (1994) sobre percepciones de las propiedades de los continuos numérico y geométrico entre estudiantes de secundaria y de primer año de college.

Por otro lado, hay que destacar que, en las descripciones del «residuo que queda en las manos» al final del proceso, éste es siempre muy pequeño o «infinitesimal» y a nadie le queda un trozo finito de cuerda. Tropezamos aquí con un obstáculo -el infinito aniquila cualquier objeto finito- $y$, de hecho, con el cúmulo de obstáculos relacionados con la noción de infinito y que ya han sido estudiados por varios autores como, por ejemplo, Fischbein, Tirosh y Hess (1979) y Moreno y Waldegg (1991).

\section{ACERCA DEL CUESTIONARIO. RESULTADOS}

El primero de los dos objetivos de ntuestro trabajo consistía en someter a ensayo el propio cuestionario. Analicemos ahora los resultados obtenidos.

La primera de las preguntas nos ha proporcionado un conjunto de datos bastante rico y complejo: el estudio de los criterios de clasificación usados para agrupar los números da información muy significativa de la relevancia que se atribuye a las propiedades y características de los diversos conjuntos de números. Ha resultado ser, por tanto, una herramienta útil para la finalidad que nos proponíamos.
La segunda pregunta de nuestro cuestionario requicre, para que los datos sean significativos, que los individuos acepten (o puedan) «jugar el juego» de la abstracción en el universo semántico que les proponemos; no obstante, la pregunta ha funcionado correctamente en la gran mayoría de los casos (83\%) y podemos considerarla válida. Para los individuos atomistas, la pregunta permite dilucidar bien la percepción de la propiedad del orden no discreto, pero los continuistas pueden contestar con coherencia e ignorar tal propiedad. Profundizar la investigación sobre este punto pide, por tanto, proponer algún otro tipo de actividad que nos permita, por una parte, estudiar las relaciones que los individuos establecen entre la recta y el continuo numérico, y, por otra parte, analizar las percepciones de las propiedades del continuo numérico en sí mismo, como conjunto provisto de estructura de orden no discreto.

La tercera pregunta del cuestionario, scguramente a causa del nivel de formación matemática de los individuos encuestados, no nos ha permitido difucidar las percepciones de la propiedad de completitud y, por lo tanto, sería necesario validarla con una muestra de individuos con niveles superiores de formación. En cambio, sí que creemos que proporciona resultados significativos respecto de las percepciones de las propiedades del infinito; en efecto, en ningún caso la descripción del residuo de cuerda corresponde a un objeto finito, sino que, como ya hemos señalado más arriba, siempre es «muy pequeño» o «infinitesimal», porque el infinito, al actuar sobre un objeto finito, lo aniquila. Por lo tanto, el valor de esta pregunta se manifiesta al permitir dilucidar el grado de articulación de las «imágenes mentales" y las "propiedades que caracterizan el concepto» en el esquema conceptual de los individuos.

\section{CONCLUSIONES}

En nuestra opinión, el análisis que hemos desarrollado proporciona indicios razonables para decir que, desde el punto de vista didáctico, las «hipótesis de la intuitividad inmediata del continuo" no tienen base que las sostenga; en efecto: tal y como demuestran las investigaciones que llevaron a definir la noción de "esquema conceptual» (véase bibl. cit.), la «intuición», que se considera como proceso inductivo que ios individuos elaboran a partir de sus experiencias (Fischbein, 1987), no es más que el recurso al propio esquema conceptual, y éste se forma, en buena parte, a través de experiencias. El análisis global ha puesto de manifiesto que, en el caso de los individuos de nuestra muestra, el esquema conceptual del continuo es un agregado inconexo de imágenes y de enunciados de propiedades que han causado un comportamiento errático ante las cuestiones que proponiamos. Tampoco hemos detectado ningún tipo de nexo no trivial entre geometría y números, hecho que tiene conexión obvia con la observación de que la característica más relevante de los números resulta ser, precisamente, su forma de escritura. 


\section{UNA CUESTIÓN ABIERTA}

Durante el desarrollo de esta investigación, hemos planteado la siguiente cuestión:

Hemos encontrado que en los esquemas conceptuales estudiados, las imágenes mentales, «partes del esquema conceptual del continuo", tienen propiedades que son lógicamente incoherentes con «las propiedades que caracterizan el concepto». Prescindiendo del hecho de que hemos estudiado individuos no expertos, nos preguntamossi los esquemas conceptuales de individuos «expertos» en un cierto concepto matemático pueden flegar a ser un conjunto de representaciones mentales desprovisto de cualquier incoherencia lógica. Si la respuesta a esto es negativa, entonces cabe preguntarse sobre la localización de lat diferencia entre un individuo «experto» y uno que no lo es; tal diferencia deberá residir en eI modo como un individuo experto trata con las diversas componentes de su esquema conceptual. Por tanto, resultará del mayor interés el estudio del proceso de coordinación de conocimientos que hacen los individuos a medida que adquieren competencia en un concepto $y$, eventualmente, hlegan a ser expertos en él.

\section{REFERENCIAS BIBLIOGRÁFICAS}

AZCÁRATE: GIMÉNEZ, C. (1990). La velocidad: introdución al concepto de derivada. Tesis doctoral, Bellaterra.

BACHELARD, G. (1938). La formation de l'esprit scientifique París: J. Vrin.

BARWISF, J y ETCHEMENDY, J. (1991). Visual Information and Valid Reasoning. Visualization in Teaching and Learning Mathematics, Zimmermann, Walter y Cunningham, Steve (eds.), pp. 9-24. Washington: The Mathematical Association of America.

BLISS, J. y OGBORN, J. en colaboración con GRIZE, F. (1979). L'anàlisi de dades qualitatives. European Joumal of Science Education, Vol. I(4), pp. 427-440.

B1,UMENTHAL, L. M., (1961). A Modern View of Geometry. Nueva York: Dover Publications, Inc.

BROUSSEAU, G., (1981). Problèmes de didactique des décimaux. Recherches en Didactique des Mathématiques, Vol. 2(3), pp. 37-127.

BROUSSEAU, (3. (1983). Les obstacles episténologiques et les problemes en mathématiques. Recherches en Didactique des Mathématiques. Vol. 4(2), pp. 165-198.

\section{NOTAS}

"Este artículo tiene como origen la iesis de maestría presentada por el autor bajo la dirección de la Dra. Carmen Azcárate, en el marco del Programa de Doctorado «Didàctica de les Matemàtiques i les Ciències», Universitat Autònoma de Barcelona.

'Creemos que Mainzer (1988) proporciona una reseña excelente de las diversas construcciones del cuerpo $R$ : cortadiras de Dedekind, sucesiones fundamentales o de Cauchy (Cantor y Méray) y encuje de intervalos cerrados (Weierstrass y Bachmann).

2 Véase, por ejemplo, Blumenthal (1961), cap. V.

3 Sobre este punto, véanse Barwise y Etchemendy (1991), y Tall (199lb).

"Por «recta graduada» entendemos el objeto que resulta de etiquetar dos puntos distintos de una recta geométrica con los números 0 y 1 .

${ }^{5}$ Textualmente: «Procura huir de frases hechas que hayas oido en clase. Te estamos pidicndo tu opinión, sentimientos quizá, sobre los números

CAVEING, MAURICE (1982). Algunas observaciones sobre el trato que recibe el continuo en los elementos de Fuclides y en la física de Aristoteles. Pensar La Matemática, Guénard, François y Lelievre, Gilbert (eds_), pp. 17-44. Barcelona: Tusquets Fditores.

CHEVALLARD, Y. (1991). La Transposition Didacique du savoir savant at savoir enseigné, suivie de un exemple de Transposition Didactique. Grenoble: Editions La Pensée Sauvage.

CORNU, B. (1980). Interférence des modèles spontanés dans l'apprentissage de la notion de limite. Cahier du Séminaire de l'Equipe de Recherche en Didactique et Pédagogie des Mathematiques, 8, pp. 57-83.

CORNU, B. (1982). Quelques obstacles à l'apprentissage de la notion de limite. Cahier du Séminaire de l'Equipe de Re. cherche en Didactique et Pédagogie des Mathematiques, 34 , pp. $235-268$.

CORNU, B. (1991). Limits, Advanced Mathematical Thinking, Tall, D. (ed.), pp. 153-166. Dordrecht: Kluwer Academic Publishers. 
DEDEKIND, R. (1901). Continuity and Irrational Numbers. Essays on the Theory of Numbers, pp. 1-27. Nueva York: Dover Publications, Inc.

DREYFUS, T et al. (1990). Advanced Mathematical Thinking. Mathematics and Cognition, Nesher, P.y Kilpatrick, J. (eds.), pp. 113-134. Cambridge: Cambridge University Press.

FISCHBEIN, E. (1987). Intuition in Science and Mathematics. Dordrecht: Kluwer Academic Publishers.

FISCHBEIN, E., TIROSH, D. y HESS, P. (1979). The Intuition of Infinity, Educational Studies in Mathematics. Vol, 10, pp. 3-40.

FISCHBEIN, E, IEHIAM, R. y COHEN, D. (1994). The Irrational Numbers and the Corresponding Epistemological Obstacles. Proceed of the XVIII International Conference for the Psychology of Mathematics Education, da Ponte, J. P. y Matos, I. F. (eds.), Vol. 2, pp. 352-359. Lisboa: Program Committee of the 18 th PME Conf.

FOWLER, D.H. (1985). 400 Years of Decimal Fractions y 400.25 Years of Decimal Fractions. Mathematics Teaching, $110 / 11 \mathrm{~L}$, pp. $20-21$ y $30-31$.

HIEBERT, J. y WEARNE, D. (1986). Procedures over Concepts: the Acquisition of Decimal Number Knowledge. Conceptual and Procedural Knowledge: The Case of Mathematics, Hiebert, James (ed.), pp. 199-223. Hillsdale NJ: Lawrence Eribatm Associates.

HILBERT, D. (1930). Lesfondements de la Géométrie, Rossier, Paul (ed.). París: Dunod.

LANG, S. (1985). Math! Encounters with High School Students Nueva York: Springer-Verlag.

LIEBECK, P. (1985), Are Fractions Numbers? Mathematics Teaching, 111, pp. 32-34.

MAINZER, K. (I988). Real Numbers. Numbers, Ewing, J.H (ed.), pp. 27-53. Nueva York: Springer-Verlag.

MANSFIELD, H. (1985). Points, Lines, and Their Representations. For the Learning of Mathematics, Vol. 5 (3), pp. 2-6.

MORENO, L.E. y WALDEGG, G. (1991). The Conceptual Evolution of Actual Mathematical Infinity. Educational Studies in Mathematics, Vol. 22, pp. 211-231
ROBINET, J. (1983). Une expérience d'ingénicrie didactique sur la notion de limite de fonction. Recherches en Didactique des Mathematiques, Vol. 4 (3), pp. 223-292.

ROBINET, J. (1986). Les réels: quels modèles en ont les éléves? Educational Studies in Mathematics, Vol. 17, pp. 359-386.

SFARD, A. (1991). On the Dual Nature of Mathematical Conceptions: Reflections on Processes and Objects as Different Sides of the Same Coin. Educational Studies in Mathematics, Vol. 22, pp. 1-36.

SIERPINSKA, A. (1985). Obstacles epistémologiques relatifs a la notion de limite. Recherches en Didactique des Mathématiques, Vol. 6 (1), pp. 5-67.

SIERPINSKA, A. (1987). Humanities Students and Epistemological Obstacles Related to Limits. Educational Studies in Mathematics, Vol. 18, pp. 371-397.

STEINER, R., (1984). Teaching About the Real Numbers. American Mathematical Monthly, Vol 91 (3), pp. 202-203.

TALL, D. (1991a). The Psichology of Advanced Mathematical Thinking. Advanced Malhematical Thinking, Tall, D. (ed.), pp. 3-21. Dordrecht: Kluwer Academic Publishers.

TALL, D., (1991b). Intuition and Rigour: The Role of Visualization in the Calculus. Visualization in Teaching and Learning Mathematics, Zimmermann, W. y Cunningham, S. (eds.), pp. 105-119. Washington: The Mathematical Association of America.

VINNER, S. (1983). Concept Definition, Concept Image and the Notion of Function. International Journal for Mathematical Education in Science and Technology. Vol. 14(3), pp. 293305 .

VINNER, S. (1991). The Role of Definitions in the Teaching and Learning of Mathematics. Advanced Mathematical Thinking, Tall, D. (ed.), pp. 65-81. Dordrecht: Kluwer Academic Publishers.

VINNER, S. y DREYFUS, T. (1989). Images and Definitions for the Concept of Function. Joumal For Research in Mathematics Education, Vol. 20(4), pp. 356-366. 